\title{
KAZHDAN-LUSZTIG POLYNOMIALS FOR HERMITIAN SYMMETRIC SPACES
}

\author{
BRIAN D. BOE
}

\begin{abstract}
A nonrecursive scheme is presented to compute the KazhdanLusztig polynomials associated to a classical Hermitian symmetric space, extending a result of Lascoux-Schützenberger for grassmannians. The polynomials for the exceptional Hermitian domains are also tabulated. All the KazhdanLusztig polynomials considered are shown to be monic.
\end{abstract}

1. Introduction. A great deal of work has recently been accomplished concerning the representation theory of groups of Hermitian type. In particular, the structure of the holomorphically induced representations having regular integral infinitesimal character is now quite well understood $[\mathbf{2}, \mathbf{6}, \mathbf{8}]$. Results in this context have proven to be significantly more tractable than in more general situations. Thus it is reasonable to expect that there should be a simple way to compute Kazhdan-Lusztig (K-L) polynomials for holomorphically induced modules. And, in fact, Lascoux-Schützenberger [11] did discover a nonrecursive scheme to compute these polynomials for $\mathrm{SU}(p, q)$. The aim of the present paper is to extend their techniques to include the other "interesting" classical Hermitian symmetric cases. (The meaning of the word "interesting" will be made clear below.)

(It should be pointed out that Enright-Shelton [8] have already obtained one generalization of the work of [11]. Namely, they obtained the analogous simple recursion formulas satisfied by the K-L polynomials, as well as a description of each coefficient in terms of "chains" of positive roots. However, our aim is to obtain the $\mathrm{K}-\mathrm{L}$ polynomials as generating functions of certain labelled trees, in keeping with the spirit of $[\mathbf{1 1}]$.)

The Hermitian symmetric pairs fall into five infinite families and two exceptional cases, as enumerated in (1.1) below. The structure of the categories for HS.2 and HS.4 is very simple, and has been known for some time [3]. In particular, there is a very simple description of the K-L polynomials, which we include for completeness. Similarly, the socle filtrations for HS.6 and HS.7 have now been worked out [6], from which it is straightforward to deduce the K-L polynomials. The results of these computations are also included in the present paper. As mentioned earlier, the polynomials for HS.1 were described in [11]. Thus there remains only the problem of their description in the cases HS.3 and HS.5. It is to this end that the main portion of the paper is devoted. In the final section, we apply our results to show that the K-L polynomials considered are all monic. And, in the cases HS.3 and HS.5, we identify those K-L polynomials having the maximum possible degree.

Received by the editors June 17, 1987. Presented at A.M.S. Meeting \#828, Logan, Utah, October 10, 1986.

1980 Mathematics Subject Classification (1985 Revision). Primary 17B10; Secondary 22E47.

Supported in part by NSF grant DMS-85-13467. 
ACKNOWLEDGMENTS. The author would like to thank the referee for pointing out a deficiency in the original definition of the trees $A(w)$, and for clarifying the statement of one of the lemmas. This paper was written at the University of California at Berkeley; the author gratefully acknowledges their support.

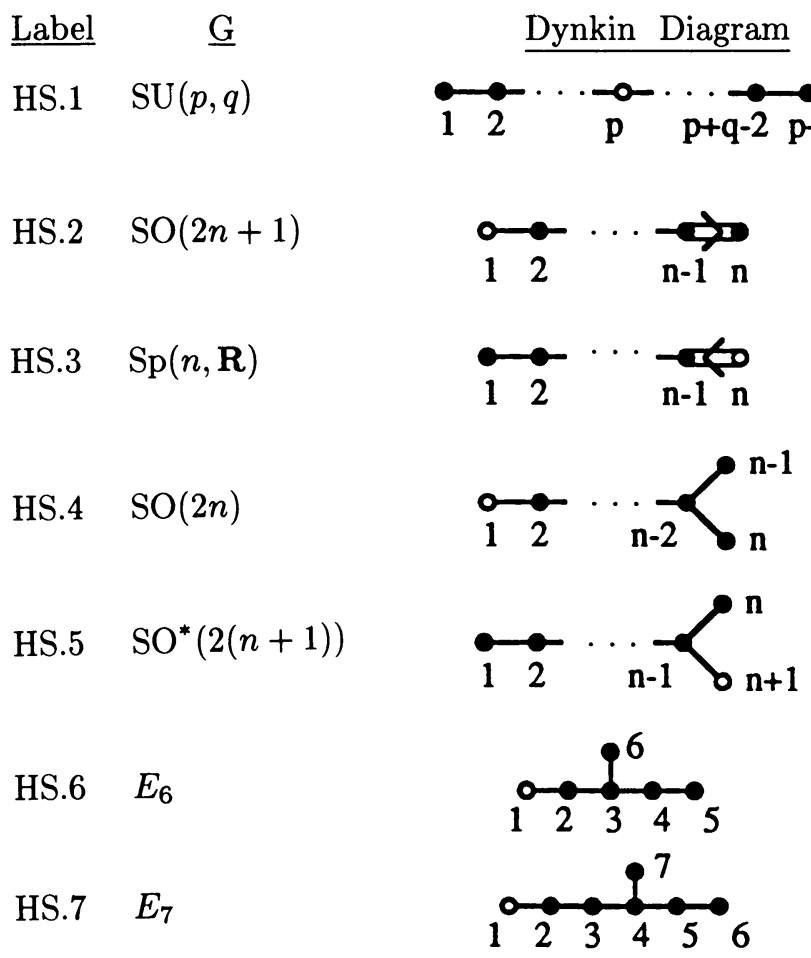

2. Preliminaries. Let $G$ be one of the groups in (1.1). Associated to a Cartan decomposition of the complexified Lie algebra, $\mathfrak{g}=\mathfrak{k}+\mathfrak{s}$, we obtain, in the usual way, a parabolic subalgebra $\mathfrak{p}=\mathfrak{k}+\mathfrak{s}^{+}$. Let $\Delta^{+}$and $\Delta_{c}^{+}$denote compatible systems of positive roots for $\mathfrak{g}$ and $\mathfrak{k}$, respectively. Let $\rho$ be one-half the sum of the positive roots for $\mathfrak{g}, W$ the Weyl group, and $W^{K}=\left\{w \in W \mid w^{-1} \Delta_{c}^{+} \subseteq \Delta^{+}\right\}$. We introduce the Bruhat order on $W$ for which the identity element is maximal. Then if $M_{w}$ denotes the Verma module with highest weight $w \rho-\rho$, we have $M_{y} \subseteq M_{w} \Leftrightarrow y \leq w$. The subset $W^{K}$ inherits a Bruhat order from $W$; moreover, the "arrow relations" generating the order on $W^{K}$ in the Hermitian symmetric cases are generated by simple root reflections (on the right). We write

$$
y \stackrel{i}{\rightarrow} w \text { means } y=w s_{i} \text { and } l(y)=l(w)+1 ; \text { here } s_{i}=s_{\alpha_{i}} .
$$

Finally, let $P_{y, w}(u)$ be the Kazhdan-Lusztig polynomials:

$$
P_{y, w}(u)=\sum_{i \geq 0} u^{i} \operatorname{dim} \operatorname{Ext}^{l(y)-l(w)-2 i}\left(M_{y}, L_{w}\right), \quad y, w \in W,
$$

where $L_{w}$ is the unique irreducible quotient of $M_{w}$.

Our aim is to compute $P_{y, w}$ for $y, w \in W^{K}$. It is known that these polynomials compute the formal characters of generalized Verma modules [4, 7]. Precisely, if 
$V_{w}$ denotes the generalized Verma module for the pair $(\mathfrak{g}, \mathfrak{p})$ having highest weight $w \rho-\rho\left(w \in W^{K}\right)$, then

$$
\operatorname{ch} L_{w}=\sum_{y \leq w, y \in W^{K}}(-1)^{l(y)-l(w)} P_{y, w}(1) \operatorname{ch} V_{y} .
$$

3. The case HS.3. Throughout this section, we will assume $G=\operatorname{Sp}(n, \mathbf{R})$. The first step is to identify concretely the set $W^{K}$, which is well known (cf. [9]). The " $\alpha \beta$-notation" introduced by Lascoux-Schützenberger for $\mathrm{SU}(p, q)$ can be adapted to the present context, as follows. View the elements of $W$ as signed permutations of $(n, n-1, \ldots, 1)$. Then the elements of $W^{K}$ can be characterized as those elements $\underline{a}=\left(a_{n}, a_{n-1}, \ldots, a_{1}\right)$ of $W$ satisfying:

$$
a_{n}>a_{n-1}>\cdots>a_{1} .
$$

To each such element $\underline{a}$ associate a word $w=w_{n} w_{n-1} \cdots w_{1}$ in two symbols $\alpha$ and $\beta$ by setting

$$
w_{i}=\beta \text { if }+i \text { appears in } \underline{a}, \quad w_{i}=\alpha \text { if }-i \text { appears in } \underline{a} .
$$

Thus, the elements of $W^{K}$ consist of all possible words $w_{n} w_{n-1} \cdots w_{1}$ in $\alpha$ and $\beta$. We shall also require an identification of $w \in W^{K}$ as a path in $\mathbf{Z} \times \mathbf{Z}$, starting at the origin: each $\alpha$ factor in $w$ corresponds to a segment $(m, n) \rightarrow(m+1, n-1)$, and each $\beta$ factor to a segment $(m, n) \rightarrow(m+1, n+1)$. Then $y \leq w$ in the Bruhat order if and only if $\operatorname{path}(y)$ lies below $\operatorname{path}(w)$.

Given any word $x=x_{m} \cdots x_{1}$ in $\alpha$ and $\beta$, define $|x|=m,|x|_{\alpha}=\#\left\{i: x_{i}=\alpha\right\}$, and similarly for $|x|_{\beta}$.

The arrow relations on $W^{K}$ are given as follows (notation as in (2.1)):

$$
\begin{aligned}
& \text { for } 1 \leq i \leq n-1: \quad y=w^{\prime} \alpha \beta w^{\prime \prime}, \quad w=w^{\prime} \beta \alpha w^{\prime \prime}, \quad \text { with }\left|w^{\prime}\right|=i-1 \\
& \text { for } i=n: \quad y=w^{\prime} \alpha, \quad w=w^{\prime} \beta, \quad \text { with }\left|w^{\prime}\right|=n-1 .
\end{aligned}
$$

From this, it is easy to deduce the length function on $W^{K}$ : for $w=w_{n} \cdots w_{1}$,

$$
l(w)=\sum_{w_{i}=\alpha} i .
$$

Notice that our conventions above imply that $\beta>\alpha$. For future reference we state the following lemma concerning "normalization" of K-L polynomials, which follows easily from $[10,2.3 \mathrm{~g}]$, and is proved in $[8,15.4 \mathrm{a}]$.

(3.5) Lemma. Let $G=\operatorname{Sp}(n, \mathbf{R})$, and parametrize $W^{K}$ as above. Assume that $\left|y^{\prime}\right|=\left|w^{\prime}\right|$.

(a) $P_{y^{\prime} \sigma \tau y^{\prime \prime}, w^{\prime} \xi \varsigma w^{\prime \prime}}=P_{y^{\prime} \tau \sigma y^{\prime \prime}, w^{\prime} \xi \varsigma w^{\prime \prime}}$ if $\sigma, \tau, \xi, \varsigma \in\{\alpha, \beta\}, \xi \geq \varsigma$.

(b) $P_{y^{\prime} \alpha, w^{\prime} \beta}=P_{y^{\prime} \beta, w^{\prime} \beta}$.

In particular, if $w=w^{\prime} w_{1} \cdots w_{r} w^{\prime \prime}$ with $w_{1} \geq \cdots \geq w_{r}, y=y^{\prime} y_{1} \cdots y_{r} y^{\prime \prime}$ with $\left|y^{\prime}\right|=\left|w^{\prime}\right|$, and $x=y^{\prime} x_{1} \cdots x_{r} y^{\prime \prime}$ with $\left(x_{1}, \ldots, x_{r}\right)$ a permutation of $\left(y_{1}, \ldots, y_{r}\right)$, then $P_{y, w}=P_{x, w}$. Hence, for the purposes of computing $P_{y, w}$, we may assume that $y$ is normalized with respect to $w$; i.e.,

1. for each factor $\alpha \beta$ in $y$, the corresponding factor in $w$ is also $\alpha \beta$; and,

2. if $y$ ends in $\alpha$, so does $w$. 
To define our generating functions, we require some additional terminology. First, let $Z$ be the smallest set of words in $\alpha$ and $\beta$ satisfying the conditions:

1. the empty word is in $Z$;

2. if $z \in Z$ then $\alpha z \beta \in Z$;

3. $Z$ is closed under concatenation.

$Z$ is the center of the "cycle monoid" introduced by Lascoux-Schützenberger. Note that $z \in Z \Rightarrow|z|_{\alpha}=|z|_{\beta}$. If $w=w^{\prime} \underline{\alpha} z \beta w^{\prime \prime}$ with $z \in Z$, we say that the indicated $\alpha$ and $\beta$ constitute a linked $\alpha \beta$-pair. If $w=w^{\prime} \alpha z$ with $z \in Z$, we say that the indicated $\alpha$ is a terminal $\alpha$. (Note that $w$ can contain at most one terminal $\alpha$.) If $w=w^{\prime} \underline{\alpha} z_{2 r} \underline{\alpha} z_{2 r-1} \alpha \cdots z_{3} \alpha z_{2} \alpha z_{1} \alpha z_{0}\left(r \geq 1, z_{i} \in Z\right)$ we say that the indicated $\alpha$ 's constitute a linked $\alpha \alpha$-pair. (Note that $w$ can contain several linked $\alpha \alpha$-pairs, all occurring to the left of a terminal $\alpha$.) In this way, all but at most one $\alpha$ in the expansion for $w$ is either a terminal $\alpha$ or part of a linked $\alpha \beta$-pair or a linked $\alpha \alpha$-pair. If there is such an $\alpha$ unaccounted for we say that it is extra. (Note that an extra $\alpha$ must be unique and must occur to the left of all the linked $\alpha \alpha$-pairs in w.)

We now introduce a rooted, directed tree $A(w)$ associated to each $w \in W^{K}$. (By convention, the root of $A(w)$ is at the top.) Each linked $\alpha \beta$-pair, $\alpha \alpha$-pair, and terminal $\alpha$ corresponds to an edge in $A(w)$. We shall refer to the edge associated to a given linked $\alpha \beta$-pair as edge $(\alpha \beta)$, etc. The attachment of edges is defined inductively using the following rules. Let $z$ denote an element of $Z$.

1. $A(\beta w)=A(w)$. (In particular, $A(\beta \cdots \beta)$ is the empty tree.)

2. To form $A(z w)$, join the trees for $A(z)$ and $A(w)$ at their roots.

3. To form $A(\underline{\alpha} z \underline{\beta})$, attach an edge above the root of $A(z)$.

4. To form $A(\underline{\alpha} z)$, attach an edge above the root of $A(z)$.

5. To form $A(\underline{\alpha} z \underline{\alpha} w)$ (where the indicated $\alpha$ 's form a linked $\alpha \alpha$-pair), attach an edge above the root of $A(z w)$.

6. $A(\underline{\alpha} w)=A(w)$ if the indicated $\alpha$ is extra.

In addition, certain other information must be encoded along with the tree. First, each edge corresponding to a terminal $\alpha$ or linked $\alpha \alpha$-pair should be marked with a "plus" sign. Second, suppose $w=w^{\prime} z_{2 r+1} \alpha z_{2 r} \alpha \cdots z_{1} \alpha z_{0}$ (as in the definition of linked $\alpha \alpha$-pair), with $z_{i} \in Z$ and $r \geq 0$ (and $z_{2 r+1}$ positive and maximal). Set $w^{\prime \prime}=\alpha z_{2 r} \alpha \cdots z_{1} \alpha z_{0}$ and $z=z_{2 r+1}$, so that $w=w^{\prime} z w^{\prime \prime}$. Also write $z=x_{s} x_{s-1} \cdots x_{1}$ with $x_{i} \in Z$ such that $x_{i}$ cannot be decomposed further into a product of (nonempty) elements of $Z(i=1, \ldots, s)$. Then $A\left(x_{i}\right)$ contains a unique "maximal edge," corresponding to a linked $\alpha \beta$-pair. And $A\left(w^{\prime \prime}\right)$ contains a unique maximal edge, corresponding to a linked $\alpha \alpha$-pair or terminal $\alpha$. View $A\left(x_{i}\right) \subseteq A(w), A\left(w^{\prime \prime}\right) \subseteq A(w)$. We say that the maximal edge of $A\left(x_{i}\right)$ (resp. $\left.A\left(w^{\prime \prime}\right)\right)$ immediately precedes the maximal edge of $A\left(x_{i+1}\right)\left(\operatorname{resp} . A\left(x_{1}\right)\right), 1 \leq i<s$. This relation can be indicated in $A(w)$ by drawing a dotted arrow from each such edge to the edge it immediately precedes. (See example below.)

Suppose $y, w \in W^{K}, y \leq w$. Each "minimal edge" of $A(w)$ corresponds either to a factor $\alpha \beta$ in $w$ (called a trough of path $(w)$ ), or to a terminal $\alpha$ at the right end 
of $w$ (called a half-trough). The capacity of a trough (or half-trough) is defined to be one-half the vertical distance from the bottom of the trough to path $(y)$. That is, if $w=w^{\prime} \alpha \beta w^{\prime \prime}$ and $y=y^{\prime} \sigma \tau y^{\prime \prime}$ with $\left|y^{\prime}\right|=\left|w^{\prime}\right|, \sigma, \tau \in\{\alpha, \beta\}$, then the capacity of edge $(\alpha \beta)$ is

$$
\operatorname{cap}(\alpha \beta)=\left|y^{\prime} \sigma\right|_{\alpha}-\left|w^{\prime} \alpha\right|_{\alpha} .
$$

And if $w=w^{\prime} \alpha$ and $y=y^{\prime} \sigma$, then the capacity of edge $(\alpha)$ is

$$
\operatorname{cap}(\alpha)=|y|_{\alpha}-|w|_{\alpha}
$$

The capacity of $w$ with respect to $y$ is the collection of capacities of all the minimal edges of $w$. These integers can be attached to the ends of the corresponding edges of $A(w)$; denote by $A(w / y)$ the tree equipped with these capacities.

(3.9) Example. Put

$$
w=\beta \alpha \alpha \beta \alpha \alpha \alpha \alpha \alpha \beta \beta \alpha \beta \alpha, \quad y=\alpha \alpha \alpha \alpha \alpha \alpha \alpha \alpha \alpha \beta \alpha \alpha \beta \alpha
$$

The linked pairs and terminal $\alpha$ in $w$ can be indicated with parentheses:

$$
w=\beta(\alpha(\alpha \beta) \alpha)(\alpha \alpha)(\alpha(\alpha \beta) \beta)(\alpha \beta)(\alpha) .
$$
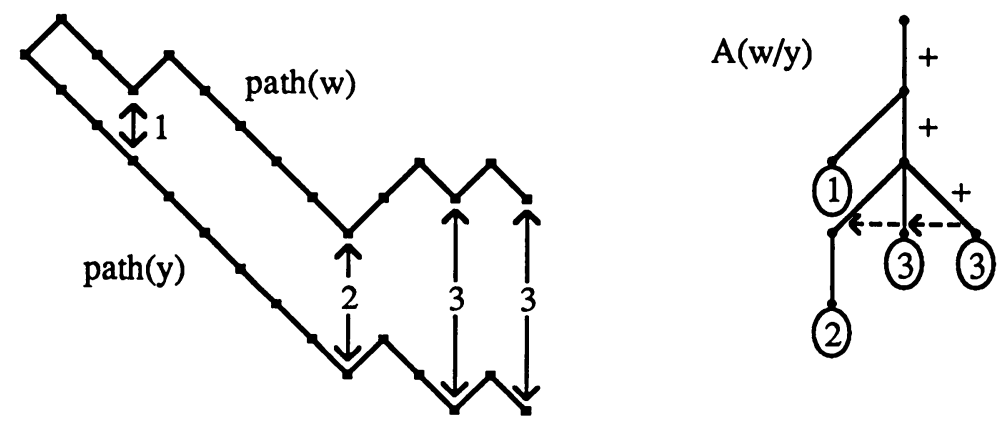

A labelling $v$ of the diagram $A(w / y)$ is a labelling of each edge with a nonnegative integer, subject to the following restrictions.

1. The label on each minimal edge is less than or equal to its capacity.

2. The integers are nonincreasing from bottom to top.

3. The integer attached to any edge equipped with a "plus" sign must be even.

4. If the label on an edge is less than or equal to the labels on all "preceding" edges, then the former must be even.

The weight $|v|$ of a labelling $v$ is the sum of the labels on all the edges. 
The generating function of the tree $A(w / y), y \leq w$, is the polynomial $Q_{y, w}(u)=$ $\sum u^{|v|}$, where the sum is taken over all labellings $v$ of $A(w / y)$. We define $Q_{y, w}(u)=$ 0 if $y \$ w$.

(3.11) EXAMPLE. Let $w=\beta \beta(\alpha \alpha)(\alpha(\alpha \beta) \beta)(\alpha), y=\alpha \alpha \alpha \alpha \alpha \alpha \beta \alpha \alpha$. Then $A(w / y)$ and its allowed labellings are as follows.
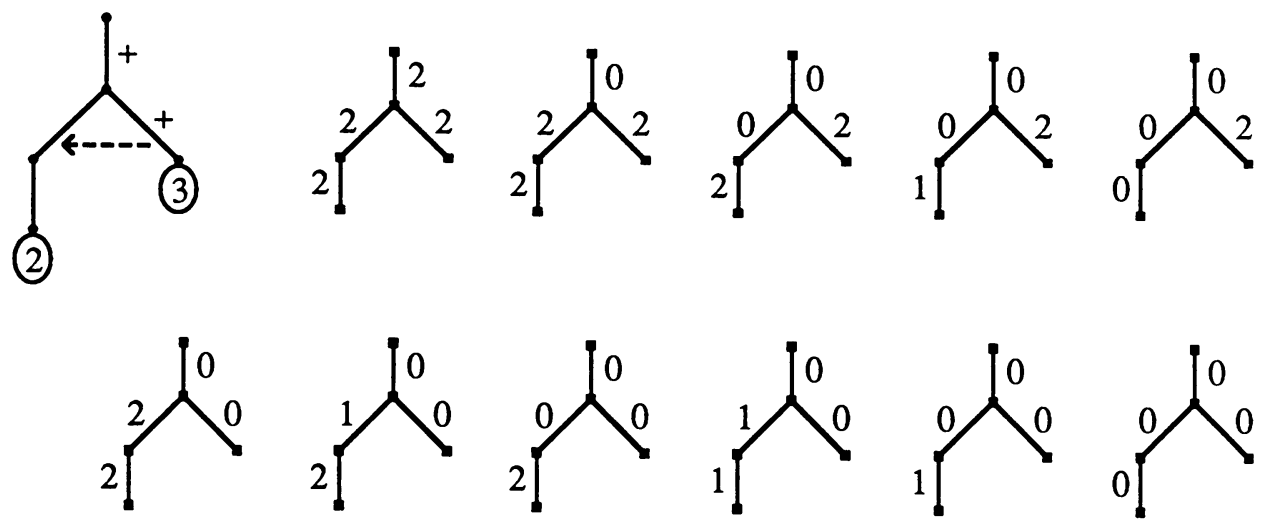

Hence

$$
\begin{aligned}
Q_{y, w}(u) & =u^{8}+u^{6}+u^{4}+u^{3}+u^{2}+u^{4}+u^{3}+u^{2}+u^{2}+u+1 \\
& =u^{8}+u^{6}+2 u^{4}+2 u^{3}+3 u^{2}+u+1
\end{aligned}
$$

Note the presence of

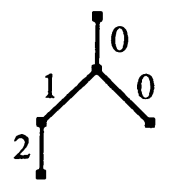

but the absence of

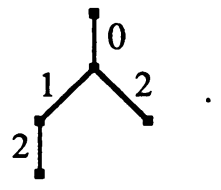


Similarly, in Example (3.9), allowed labellings include
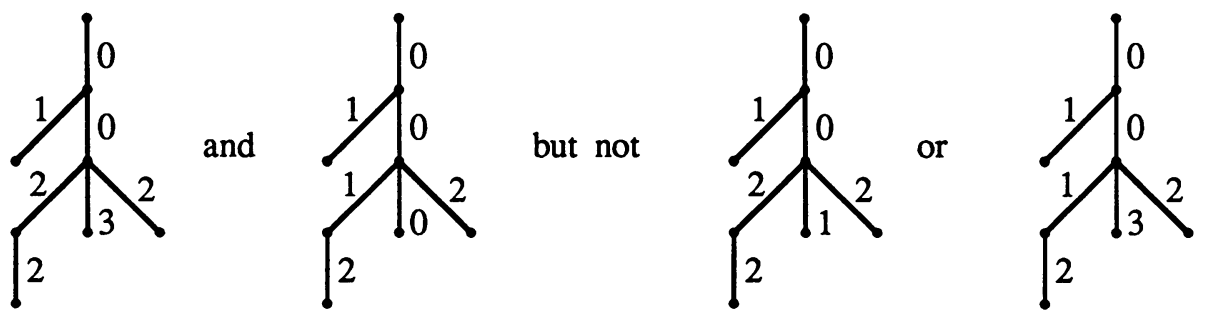

(3.12) REMARK. It is clear that the polynomials $Q_{y, w}$ satisfy the "normalization conditions" of (3.5): the indicated changes in $y$ do not change any of the capacities. Hence, for computing $Q_{y, w}$, we may also assume $y$ is normalized with respect to $w$ (cf. 3.6).

We can now state our main theorem in the case HS.3.

(3.13) THEOREM. Let $G=\operatorname{Sp}(n, \mathbf{R})$, and let $Q_{y, w}(u)$ be the polynomial defined above. Then $Q_{y, w}(u)=P_{y, w}(u)$ for all $y, w \in W^{k}$.

The proof will depend on the following recursion relations satisfied by the polynomials $Q_{y, w}$.

(3.14) Proposition. (a) Let $y=y^{\prime} \alpha \beta y^{\prime \prime}, w=w^{\prime} \alpha \beta w^{\prime \prime}\left(\left|y^{\prime}\right|=\left|w^{\prime}\right|\right)$, and put $c=\left|y^{\prime}\right|_{\alpha}-\left|w^{\prime}\right|_{\alpha}$ (the capacity of the distinguished minimal edge). Then

$$
Q_{y, w}=Q_{y^{\prime} \beta \alpha y^{\prime \prime}, w^{\prime} \alpha \beta w^{\prime \prime}}+u^{c} Q_{y^{\prime} y^{\prime \prime}, w^{\prime} w^{\prime \prime}}
$$

(b) Let $y=y^{\prime} \sigma \alpha, w=w^{\prime} \tau \alpha, \sigma, \tau \in\{\alpha, \beta\}$, and put $c=|y|_{\alpha}-|w|_{\alpha}$ (the capacity of the distinguished minimal edge). Then

$$
Q_{y, w}=\left\{\begin{array}{l}
Q_{y^{\prime} \sigma \beta, w^{\prime} \tau \alpha}+u^{c} Q_{y^{\prime}, w^{\prime}}, \quad c \text { even }, \\
Q_{y^{\prime} \sigma \beta, w^{\prime} \tau \alpha}, \quad c \text { odd. }
\end{array}\right.
$$

PROOF OF (3.13). Let us assume the recursion relations of (3.14). We claim that these are precisely the relations of [8, Theorem 15.4] satisfied by the K-L polynomials. For $y$ normalized with respect to $w$, the latter relations expressed in our notation are as follows. (Note that Enright-Shelton use the opposite Bruhat order to ours.)

(i) $P_{y^{\prime} \alpha \beta y^{\prime \prime}, w^{\prime} \alpha \beta w^{\prime \prime}}=P_{y^{\prime} \beta \alpha y^{\prime \prime}, w^{\prime} \alpha \beta w^{\prime \prime}}+u^{r} P_{y^{\prime} y^{\prime \prime}, w^{\prime} w^{\prime \prime}}$ where $2 r=\left[l(y)-l\left(y^{\prime} y^{\prime \prime}\right)\right]-\left[l(w)-l\left(w^{\prime} w^{\prime \prime}\right)\right]$;

$$
\begin{aligned}
& \text { (ii) } P_{y^{\prime} \sigma \alpha, w^{\prime} \tau \alpha}=P_{y^{\prime} \sigma \beta, w^{\prime} \tau \alpha}+u^{r} P_{y^{\prime}, w^{\prime}} \quad \text { if }|y|_{\alpha} \equiv|w|_{\alpha}(\bmod 2) \\
& \text { where } 2 r=\left[l(y)-l\left(y^{\prime}\right)\right]-\left[l(w)-l\left(w^{\prime}\right)\right] \text {; } \\
& \text { (iii) } \quad P_{y^{\prime} \sigma \alpha, w^{\prime} \tau \alpha}=P_{y^{\prime} \sigma \beta, w^{\prime} \tau \alpha} \quad \text { if }|y|_{\alpha} \not \equiv|w|_{\alpha}(\bmod 2) .
\end{aligned}
$$

Thus to prove the claim, it remains to check that $r=c$. But in case (i), it follows from (3.4) that

$$
l\left(y^{\prime} \alpha \beta y^{\prime \prime}\right)-l\left(y^{\prime} y^{\prime \prime}\right)=\left(\left|y^{\prime \prime}\right|+2\right)+2\left|y^{\prime}\right|_{\alpha},
$$


and similarly for $w$. Thus

$$
2 r=2\left(\left|y^{\prime}\right|_{\alpha}-\left|w^{\prime}\right|_{\alpha}\right)=2 c .
$$

In case (ii),

$$
l(y)-l\left(y^{\prime}\right)=1+2 \delta_{\sigma \alpha}+2\left|y^{\prime}\right|_{\alpha} \quad \text { (Kronecker delta), }
$$

and similarly for $w$. Thus

$$
2 r=2\left[\left(\left|y^{\prime}\right|_{\alpha}+\delta_{\sigma \alpha}\right)-\left(\left|w^{\prime}\right|_{\alpha}+\delta_{\tau \alpha}\right)\right]=2\left(|y|_{\alpha}-|w|_{\alpha}\right)=2 c .
$$

That $Q_{y, w}=P_{y, w}$ now follows by induction on the rank $n$, and induction on $l(y)-l(w)$. Q.E.D.

(3.16) LEMmA. Let $y, w \in W^{K}$ with $y \leq w$. In each case (a)-(e) below, $A(w)$ has a distinguished minimal edge (indicated by underlining the corresponding factor in $w)$. Assume that if $w=w^{\prime} \alpha \beta w^{\prime \prime}$ then $y=y^{\prime} \alpha \beta y^{\prime \prime}$ with $\left|y^{\prime}\right|=\left|w^{\prime}\right|$, and if $w=w^{\prime} \underline{\alpha}$ then $y=y^{\prime} \alpha$. Let $c$ be the capacity of the distinguished minimal edge. Let $z$ denote a nonempty element of $Z$.

(a) Let $w=\cdots z \alpha \beta \cdots$. Then $z$ contains a factor $\alpha_{1} \beta_{1}$ such that $\operatorname{cap}\left(\alpha_{1} \beta_{1}\right) \leq c$.

(b) Let $w=\cdots z \underline{\alpha}$. Then $z$ contains a factor $\alpha_{1} \beta_{1}$ such that $\operatorname{cap}\left(\alpha_{1} \beta_{1}\right) \leq c$.

(c) Let $w=\cdots \alpha z \beta \underline{\alpha}$. Then $z$ contains a factor $\alpha_{1} \beta_{1}$ such that $\operatorname{cap}\left(\alpha_{1} \beta_{1}\right) \leq c$.

(d) Let $w=\cdots \alpha z \alpha \underline{\alpha}$. Then $z$ contains a factor $\alpha_{1} \beta_{1}$ such that $\operatorname{cap}\left(\alpha_{1} \beta_{1}\right) \leq c+1$.

(e) Let $w=\cdots \alpha \beta z \alpha_{0} \cdots$, where $\alpha_{0}$ is either a terminal $\alpha$ or the left member of a linked $\alpha \alpha$ pair. Then $z$ contains a factor $\alpha_{1} \beta_{1}$ such that $\operatorname{cap}\left(\alpha_{1} \beta_{1}\right) \leq c$. Moreover, edge $\left(\alpha_{1} \beta_{1}\right)$ lies below (or is) an edge which immediately precedes edge $(\alpha \beta)$.

PROOF. For (a)-(d), observe that $z=\cdots \alpha_{1} \beta_{1} \beta \cdots \beta$. Considering the paths of $w$ and of $y$ (and recalling that the factor in $y$ corresponding to the underlined $\alpha$ in $w$ is also $\alpha$ ), it is clear that the capacity of the $\alpha_{1} \beta_{1}$-trough is at most $c$ in cases (a) $-(\mathrm{c})$, and at most $c+1$ in case $(\mathrm{d})$.

For $(\mathrm{e})$, observe that $z=\alpha \alpha \cdots \alpha_{1} \beta_{1} \cdots$. As above, $\operatorname{cap}\left(\alpha_{1} \beta_{1}\right) \leq c$. Also, writing $z=x_{s} \cdots x_{1}$ as in the definition of "immediate predecessor," it is obvious that $\alpha_{1} \beta_{1}$ occurs in $x_{s}$. Hence edge $\left(\alpha_{1} \beta_{1}\right)$ lies below (or is) the top edge of $A\left(x_{s}\right)$, which immediately precedes edge $(\alpha \beta)$. Q.E.D.

PROOF OF (3.14). Given a minimal edge of $A(w / y)$ having capacity $c$, the labellings of $A(w / y)$ split into two families, according to whether the label on this minimal edge is $\langle c$ or $=c$. Evidently the first family has generating function given by the first term on the right-hand side of each recursion relation in (3.14). Thus we must show that the generating function, say $Q_{c}(u)$, of the second family, is equal to the second term (if present) in the recursion relations.

For the proof, it will be convenient to have a generalization of the notion of capacity to nonminimal edges of $A(w / y)$. If edge $(\mu)$ is such an edge, define its capacity, $\operatorname{cap}(\mu)$, to be its largest allowed label in any labelling of $A(w / y)$. Also, denote by $A^{\prime}$ the tree $A\left(w^{\prime} w^{\prime \prime}\right)$ (resp. $A\left(w^{\prime}\right)$ ) in case (a) (resp. (b)). Then $A^{\prime}$ is obtained from $A(w)$ by removing the distinguished minimal edge. If edge $(\psi)$ is any other edge of $A(w)$, we must show that $\operatorname{cap}(\psi)=\operatorname{cap}^{\prime}(\psi)$ (the capacity of edge $(\psi)$ when viewed in $A^{\prime}$ ), and that any parity restrictions on the labels assigned to edge $(\psi)$ are the same in $A^{\prime}$ as in $A(w)$.

Notice first that, in case (b) when $c$ is odd, the second family is empty, since the label on an edge corresponding to a terminal $\alpha$ must be even. 
Now assume we are in case (b) with $c$ even. It is clearly sufficient to consider edges immediately above, or preceded by, the terminal $\alpha$ edge. Suppose first that $w=\cdots \alpha_{1} z \beta_{1} \alpha, z \in Z$ (so $w^{\prime}=\cdots \alpha_{1} z$ ). Then edge $\left(\alpha_{1} \beta_{1}\right)$, which is immediately preceded by edge $(\alpha)$, becomes a terminal $\alpha$ edge in $A^{\prime}$. If $z$ is the empty word, then $\operatorname{cap}\left(\alpha_{1} \beta_{1}\right)=\operatorname{cap}^{\prime}\left(\alpha_{1}\right) \leq c$. Otherwise, Lemma 3.16 implies that $\operatorname{cap}\left(\alpha_{1} \beta_{1}\right)=$ $\operatorname{cap}^{\prime}\left(\alpha_{1}\right) \leq c$. Thus in all labellings contributing to $Q_{c}$, the label on edge $\left(\alpha_{1} \beta_{1}\right)$ is less than or equal to the label on the terminal $\alpha$ edge, hence must be even. Also, if the label on any edge preceded by edge $(\alpha)$ is less than or equal to all labels preceding it except perhaps that of edge $(\alpha)$, then in particular it is $\leq \operatorname{cap}\left(\alpha_{1} \beta_{1}\right)$, hence it $i s$ in fact $\leq c$. This takes care of any edges preceded by the terminal $\alpha$ edge.

Next suppose that $w=\cdots \alpha_{1} z^{\prime} \alpha_{2} z \alpha$. If $z$ is not empty, then again (3.16) implies that $\operatorname{cap}\left(\alpha_{1} \alpha_{2}\right)=\operatorname{cap}^{\prime}\left(\alpha_{1} \alpha_{2}\right) \leq c$. Similarly, if $z$ is empty, then $\operatorname{cap}\left(\alpha_{1} \alpha_{2}\right)=$ $\operatorname{cap}^{\prime}\left(\alpha_{1}\right) \leq c+1$ (use the lemma if $z^{\prime}$ is not empty). 'But $c+1$ is odd, so any label on this edge in $A^{\prime}$ must still be $\leq c$. This takes care of any edge above the terminal $\alpha$ edge, and completes the proof of (b).

Finally assume we are in case (a). Here we must examine the effect of removing edge $(\alpha \beta)$ on: (i) $\alpha \beta$ edges above it; (ii) a terminal $\alpha$ edge above it; (iii) edges which it precedes or is preceded by; and (iv) an $\alpha \alpha$ edge above it. We treat each of these in turn.

Case (i). $w=\cdots \alpha_{1} z \alpha \beta z^{\prime} \beta_{1} \cdots$, so that edge $\left(\alpha_{1} \beta_{1}\right)$ is attached above edge $(\alpha \beta)$. If $z$ is not empty, then it follows from Lemma 3.16 that $\operatorname{cap}\left(\alpha_{1} \beta_{1}\right)=\operatorname{cap}^{\prime}\left(\alpha_{1} \beta_{1}\right) \leq c$. Otherwise, $\operatorname{cap}\left(\alpha_{1} \beta_{1}\right)=\operatorname{cap}^{\prime}\left(\alpha_{1} \beta_{1}\right)=c$.

Case (ii). $w=\cdots \alpha_{1} z \alpha \beta z^{\prime}$, so that edge $\left(\alpha_{1}\right)$ is attached above edge $(\alpha \beta)$. As in case (i), we find that $\operatorname{cap}\left(\alpha_{1}\right)=\operatorname{cap}^{\prime}\left(\alpha_{1}\right) \leq c$.

Case (iii). Assume that the $\alpha \beta$ edge in question is preceded by at least one edge. We claim that one of these preceding edges, say edge $(\mu)$, has capacity $\leq c$ (in both $A$ and $\left.A^{\prime}\right)$. Suppose we have shown this. Then $c$ is an allowed label on edge $(\alpha \beta)$ in $A(w / y)$. (For if $c$ is $\leq$ the labels on all edges preceding edge $(\alpha \beta)$, then the label on edge $(\mu)$ must be both $=c$ and $\leq$ the labels on all edges preceding $i$, forcing $c$ to be even.) Moreover, if edge $(\psi)$ is preceded by edge $(\alpha \beta)$, then the label on edge $(\psi)$ is $\leq$ all preceding labels in $A(w / y)$ if and only if it is $\leq$ all preceding labels in $A^{\prime}$.

To prove the claim, write $w=\cdots \alpha \beta z \alpha z_{2 r} \alpha z_{2 r-1} \cdots \alpha z_{2} \alpha z_{1} \alpha z_{0}(r \geq 0)$. If $z$ is nonempty, then the edge immediately preceding edge $(\alpha \beta)$ has capacity $\leq c$, by the lemma. Otherwise, if some $z_{i}$ is nonempty for $1 \leq i \leq 2 r$, the $\alpha \alpha$-edge preceding edge $(\alpha \beta)$ has capacity $\leq c$. Otherwise, the terminal $\alpha$ edge has capacity $\leq c$, again forcing the $\alpha \alpha$ - (or terminal $\alpha$-) edge preceding edge $(\alpha \beta)$ to have capacity $\leq c$.

Case (iv). Let $w=\cdots \alpha_{1} z_{2 r} \alpha_{2} z_{2 r-1} \alpha \cdots z_{3} \alpha z_{2} \alpha z_{1} \alpha z_{0}(r \geq 1)$, where the linked $\alpha \beta$-pair in question is either part of $z_{2 r-1}$ or of $z_{2 r}$. In the former case, edge $(\alpha \beta)$ has predecessors, and the argument in case (iii) applies to show that one of these predecessors has capacity $\leq c$ (and lies below edge $\left(\alpha_{1} \alpha_{2}\right)$ ). So assume we are in the latter case; say $w=\cdots \alpha_{1} z \alpha \beta z^{\prime} \alpha_{2} z_{2 r-1} \cdots$. If $z$ or $z^{\prime}$ is nonempty, then (3.16) implies that $\operatorname{cap}\left(\alpha_{1} \alpha_{2}\right)=\operatorname{cap}^{\prime}\left(\alpha_{1} \alpha_{2}\right) \leq c$. Otherwise, an argument as in case (iii) shows that some minimal edge (besides the distinguished $\alpha \beta$-edge) below edge $\left(\alpha_{1} \alpha_{2}\right)$ has capacity $\leq c$.

This completes the proof of the proposition. Q.E.D. 
4. The case HS.5. In this section, assume $G=\operatorname{SO}^{*}(2(n+1))$. Here $W^{K}$ consists of decreasing signed permutations of $(n+1, n, \ldots, 1)$ having an even number of minus signs. We associate to such a signed permutation a word of length $n+1$ in $\alpha$ and $\beta$ by the rule (3.2). But clearly the rightmost symbol is redundant (since there must be an even number of $\alpha$ 's), so we may drop it. We may therefore parametrize $W^{K}$ exactly as in the case HS.3. There is a slight change in the arrow relations: $w^{\prime} \alpha \rightarrow w^{\prime} \beta$ corresponds to $i=n$ if $\left|w^{\prime}\right|_{\alpha}$ is odd but to $i=n+1$ if $\left|w^{\prime}\right|_{\alpha}$ is even (notation as in (2.1) and (3.3)).

For $y \leq w$ in $W^{K}$, define $A(w), A(w / y)$, and $Q_{y, w}(u)$ exactly as in the case HS.3. Then the recursion relations of (3.14) hold in HS.5. But the Enright-Shelton relations (3.15) also characterize the $P_{y, w}$ for HS.5. (Here, the formula in (3.15(iii)) arises not from a parity difference on the long simple root wall, but rather from the fact that $y s_{n} \in W^{K}$ while $w s_{n+1} \in W^{K}$ (or vice versa) when $c$ is odd; cf. the remarks in the previous paragraph.) Hence the same proof gives

(4.1) THEOREM. Let $G=\mathrm{SO}^{*}(2(n+1))$, and let $Q_{y, w}$ be the polynomial defined above for $y, w \in W^{K}$. Then $Q_{y, w}(u)=P_{y, w}(u)$.

5. The cases HS.2, HS.4. Assume that $G=\mathrm{SO}(n, \mathbf{R})$. In this case there are simple closed form descriptions of the K-L polynomials. They can be proved using Deodhar's recursion formulas [7, Proposition 3.9], or from the known socle filtrations of the generalized Verma modules [3]; cf. the remarks in $\S 6$ below.

(5.1) Proposition. (a) Let $G=\mathrm{SO}(2 n+1, \mathbf{R})$. Then $W^{K}$ is a simple chain, and $P_{y, w}(u) \equiv 1$ for all $y \leq w$ in $W^{K}$ (and $P_{y, w}(u) \equiv 0$ otherwise).

(b) Let $G=\mathrm{SO}(2 n, \mathbf{R})$. Parametrize the partially ordered set $W^{K}$ as in [5, (4.3)]: $W^{K}=\left\{w_{i} \mid 1 \leq i \leq 2 n\right\}$, with $w_{i} \leq w_{j}$ for all $i \leq j$ except that $w_{n}$ and $w_{n+1}$ are incomparable. Then

$$
P_{w_{i}, w_{j}}(u)= \begin{cases}0, & w_{i} \nless w_{j}, \\ 1+u^{j-n-1}, & n+2 \leq j \leq 2 n-1,1 \leq i \leq 2 n-j, \\ 1, & \text { otherwise. }\end{cases}
$$

6. The cases HS.6, HS.7. The K-L polynomials in the exceptional cases can be obtained as follows. Let $S_{y, w}(u)$ be the polynomials which invert (2.3); i.e. such that

$$
\operatorname{ch} V_{w}=\sum_{y \leq w, y \in W^{K}} S_{y, w}(1) \operatorname{ch} L_{y}
$$

Also let $0=V_{n(w)+1} \subset V_{n(w)} \subset \cdots \subset V_{0}=V_{w}$ be the socle filtration of $V_{w}$ with $i$ th layer $V_{i} / V_{i+1}(0 \leq i \leq n(w))$. It follows from [4 and 6] that

$$
S_{y, w}(u)=\sum_{i=0}^{n(w)} a(i, y, w) u^{(l(y)-l(w)-i) / 2}
$$

where $a(i, y, w)$ is the multiplicity of $L_{y}$ in the $i$ th layer of $V_{w}$. But these multiplicities are known [6, Tables 7.1, 7.2]. Thus the polynomials $S_{y, w}$ are known. The matrices $\left(S_{y, w}(u)\right)$ were inverted (using a symbolic manipulation computer program) to obtain the matrices of K-L polynomials. Space considerations have necessitated replacing each polynomial (except for 0 and 1) with a single symbol 
TABLE 1. Key for Tables 2, 3

\begin{tabular}{llll} 
a & $u+1$ & w & $u^{4}+2 u^{3}+2 u^{2}+2 u+1$ \\
b & $u^{2}+1$ & X & $u^{5}+u^{3}+u^{2}+u+1$ \\
C & $u^{2}+u+1$ & y & $u^{5}+u^{4}+u^{3}+u^{2}+u+1$ \\
d & $u^{3}+1$ & z & $u^{5}+u^{4}+u^{3}+2 u^{2}+u+1$ \\
e & $u^{3}+u+1$ & A & $u^{5}+2 u^{4}+2 u^{3}+2 u^{2}+2 u+1$ \\
f & $u^{3}+u^{2}+1$ & B & $u^{6}+u^{3}+u^{2}+1$ \\
g & $u^{3}+u^{2}+u+1$ & C & $u^{6}+2 u^{3}+u^{2}+u+1$ \\
\hline h & $u^{2}+2 u+1$ & D & $u^{6}+u^{4}+u^{3}+u^{2}+1$ \\
i & $u^{3}+2 u^{2}+2 u+1$ & E & $u^{6}+2 u^{4}+2 u^{3}+u^{2}+u+1$ \\
j & $u^{4}+1$ & F & $u^{6}+u^{5}+2 u^{4}+u^{3}+2 u^{2}+u+1$ \\
k & $u^{4}+u+1$ & G & $u^{7}+u^{4}+u^{3}+1$ \\
m & $u^{4}+u^{2}+1$ & H & $u^{7}+u^{4}+u^{3}+u^{2}+1$ \\
n & $u^{4}+u^{2}+u+1$ & J & $u^{7}+2 u^{4}+u^{3}+u+1$ \\
P & $u^{4}+2 u^{2}+u+1$ & K & $u^{7}+u^{5}+u^{4}+u^{3}+u^{2}+u+1$ \\
q & $u^{4}+u^{3}+1$ & L & $u^{7}+u^{6}+u^{4}+2 u^{3}+u^{2}+u+1$ \\
I & $u^{4}+u^{3}+u+1$ & M & $u^{8}+u^{4}+1$ \\
S & $u^{4}+u^{3}+u^{2}+1$ & N & $u^{8}+u^{4}+u+1$ \\
t & $u^{4}+u^{3}+u^{2}+u+1$ & P & $u^{8}+u^{4}+u^{2}+u+1$ \\
V & $u^{4}+2 u^{3}+u^{2}+u+1$ & $Q$ & $u^{8}+u^{4}+u^{3}+u^{2}+u+1$ \\
& & $R$ & $u^{8}+2 u^{4}+u^{3}+u^{2}+u+1$
\end{tabular}

TABLE 2. Kazhdan-Lusztig polynomials for $E_{6}$

\begin{tabular}{|c|c|c|c|c|c|c|c|c|c|c|c|c|c|c|c|c|c|c|c|c|c|c|c|c|c|c|c|}
\hline & 0 & & & & & & & & & & & & & & & & & & & & & & & & & & \\
\hline 0 & & & & & & & & & & & d & & & & & & 8 & & & & & & & & & & \\
\hline 1 & 0 & & & & & & o & & 8 & & 1 & c & & & b & a & c & & & & 2 & b & & & $\mathbf{f}$ & & 1 \\
\hline 2 & 0 & 0 & 1 & 1 & 1 & & & 1 & & & 1 & $\mathbf{a}$ & & & 1 & & 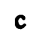 & c & b & & 1 & b & a & & f & d & 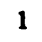 \\
\hline 3 & 0 & 0 & 0 & 1 & 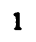 & 1 & 1 & 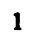 & & 1 & 1 & $\mathbf{a}$ & & & 1 & & $\mathbf{a}$ & & & & 1 & & ? & & f & & 1 \\
\hline 4 & 0 & 0 & 0 & 0 & 1 & 0 & 1 & ( & 1 & 1 & 1 & a & 1 & $\mathbf{a}$ & 1 & Q & $\mathbf{a}$ & $\mathbf{a}$ & 1 & $\mathbf{a}$ & 1 & 1 & $\mathbf{a}$ & c & f & d & 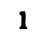 \\
\hline 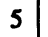 & 0 & 0 & 0 & 0 & 0 & 1 & 1 & 1 & 1 & 1 & 1 & 1 & 1 & 1 & 1 & 1 & a & 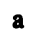 & & c & 1 & & 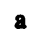 & & b & & 1 \\
\hline 6 & 0 & 0 & 0 & 0 & 0 & 0 & 1 & ( & 1 & 1 & 1 & 1 & . & 1 & 1 & 1 & a & $\mathbf{a}$ & 1 & $\mathbf{a}$ & 1 & & $\mathbf{a}$ & c & 0 & d & . \\
\hline 7 & 0 & 0 & 0 & 0 & 0 & 0 & 0 & 1 & 0 & 1 & 0 & 1 & 0 & 1 & 1 & 1 & a & $a$ & , & c & 1 & & 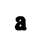 & $c$ & b & 1 & 1 \\
\hline 8 & 0 & 0 & 0 & 0 & 0 & 0 & 0 & 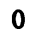 & 1 & ( & 1 & 1 & & & & & & & & $\mathbf{a}$ & 1 & & $\mathbf{a}$ & $\mathbf{a}$ & b & d & , \\
\hline 9 & 0 & 0 & 0 & 0 & 0 & 0 & 0 & 0 & 0 & 1 & 0 & 1 & & & 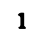 & 1 & $\mathbf{a}$ & $\mathbf{a}$ & 1 & $\mathbf{a}$ & 1 & & $\mathbf{a}$ & c & b & 1 & 1 \\
\hline 10 & 0 & 0 & 0 & 0 & 0 & 0 & 0 & 0 & 0 & 0 & 1 & 0 & 1 & 1 & 0 & 1 & 1 & & & 1 & 1 & & 1 & & b & d & 1 \\
\hline 11 & 0 & 0 & 0 & 0 & 0 & 0 & 0 & 0 & 0 & 0 & 0 & 1 & & & 1 & & 1 & & & $\mathbf{a}$ & 1 & 1 & $a$ & $\mathbf{a}$ & b & 1 & 1 \\
\hline 12 & 0 & 0 & 0 & 0 & 0 & 0 & 0 & 0 & 0 & 0 & 0 & 0 & 1 & 0 & 0 & 1 & 0 & 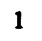 & 0 & 1 & 0 & & 1 & $\mathbf{a}$ & b & d & 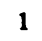 \\
\hline 13 & 0 & 0 & 0 & 0 & 0 & 0 & 0 & 0 & 0 & 0 & 0 & 0 & 0 & 1 & 0 & 1 & & & & 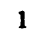 & 1 & & 1 & & b & 1 & 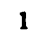 \\
\hline 14 & 0 & 0 & 0 & 0 & 0 & 0 & 0 & 0 & 0 & 0 & 0 & 0 & 0 & 0 & 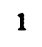 & 0 & 1 & & 1 & $\mathbf{a}$ & 1 & & $\mathbf{a}$ & $a$ & 1 & 1 & , \\
\hline 15 & 0 & 0 & 0 & 0 & 0 & 0 & 0 & 0 & 0 & 0 & 0 & 0 & 0 & 0 & 0 & 1 & 0 & 1 & 0 & 1 & 0 & 1 & 1 & $\mathbf{a}$ & b & 1 & 1 \\
\hline 16 & 0 & 0 & 0 & 0 & 0 & 0 & 0 & 0 & 0 & 0 & 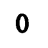 & 0 & 0 & 0 & 0 & 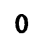 & 1 & 1 & 1 & 1 & 1 & & 1 & & 1 & 1 & 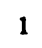 \\
\hline 17 & 0 & 0 & 0 & 0 & 0 & 0 & 0 & 0 & 0 & 0 & 0 & 0 & 0 & 0 & 0 & 0 & 0 & 1 & 0 & 1 & 0 & 1 & 1 & $\mathbf{a}$ & 1 & 1 & 1 \\
\hline 18 & 0 & 0 & 0 & 0 & 0 & 0 & 0 & & 0 & 0 & 0 & 0 & & 0 & 0 & 0 & 0 & 0 & & 1 & 1 & 1 & 1 & 1 & 1 & 1 & 1 \\
\hline 19 & 0 & 0 & 0 & 0 & 0 & 0 & 0 & 0 & 0 & 0 & 0 & 0 & 0 & 0 & 0 & 0 & 0 & 0 & 0 & 1 & 0 & 1 & 1 & 1 & 1 & 1 & 1 \\
\hline 20 & 0 & 0 & 0 & 0 & 0 & 0 & 0 & & 0 & 0 & 0 & 0 & & & 0 & 0 & 0 & 0 & 0 & 0 & 1 & 0 & 1 & 1 & 1 & 1 & 1 \\
\hline 21 & 0 & 0 & 0 & 0 & 0 & 0 & 0 & 0 & 0 & 0 & 0 & 0 & 0 & 0 & 0 & 0 & 0 & 0 & 0 & 0 & 0 & 1 & 0 & 1 & 1 & 1 & 1 \\
\hline 22 & 0 & 0 & 0 & 0 & 0 & 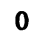 & 0 & 0 & 0 & ( & 0 & 0 & 0 & 0 & 0 & 0 & 0 & 0 & 0 & 0 & 0 & 0 & 1 & 1 & 1 & 1 & 1 \\
\hline 23 & 0 & 0 & 0 & 0 & 0 & $v$ & 0 & 0 & 0 & 0 & 0 & 0 & 0 & 0 & 0 & 0 & 0 & 0 & 0 & 0 & 0 & 0 & 0 & 1 & 1 & 1 & 1 \\
\hline 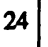 & 0 & 0 & 0 & 0 & 0 & 0 & 0 & 0 & 0 & 0 & 0 & 0 & 0 & 0 & . & - & 0 & 0 & 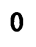 & 0 & 0 & 0 & 0 & 0 & 1 & 1 & 1 \\
\hline 25 & 0 & 0 & . & 0 & 0 & 0 & 0 & 0 & 0 & 0 & 0 & 0 & 0 & 0 & 0 & 0 & 0 & 0 & 0 & 0 & 0 & 0 & 0 & & 0 & 1 & 1 \\
\hline 26 & 0 & 0 & 0 & 0 & 0 & 0 & 0 & 0 & C & 0 & 0 & 0 & 0 & 0 & 0 & 0 & 0 & 0 & & 0 & 0 & 0 & 0 & & 0 & 0 & 1 \\
\hline
\end{tabular}


TABLE 3. Kazhdan-Lusztig polynomials for $E_{7}$

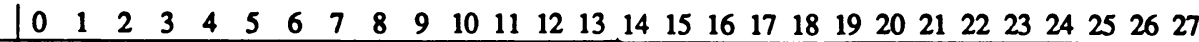

$\begin{array}{lllllllllllllllllllllllllllll}0 & 1 & 1 & 1 & 1 & 1 & 1 & 1 & a & 1 & b & a & d & c & j & e & b & 1 & k & g & a & n & f & c & t & d & g & m & r\end{array}$

$\begin{array}{lllllllllllllllllllllllllllll}1 & 0 & 1 & 1 & 1 & 1 & 1 & 1 & a & 1 & b & a & d & c & 1 & e & b & 1 & a & g & a & c & f & c & g & d & g & b & e\end{array}$

$\begin{array}{lllllllllllllllllllllllllllll}2 & 0 & 0 & 1 & 1 & 1 & 1 & 1 & a & 1 & b & a & 1 & c & 1 & a & b & 1 & a & c & a & c & b & c & g & 1 & g & b & c\end{array}$

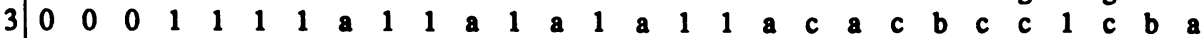

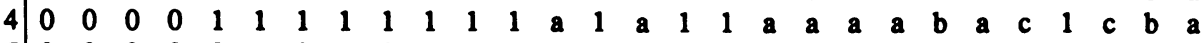

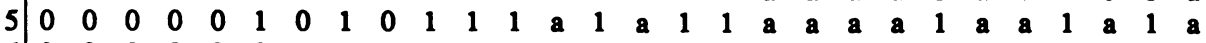

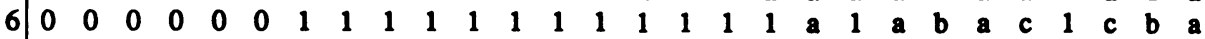

$7 \quad 0 \begin{array}{llllllllllllllllllllllllllll} & 0 & 0 & 0 & 0 & 0 & 0 & 1 & 0 & 1 & 1 & 1 & 1 & 1 & 1 & 1 & 1 & 1 & a & 1 & a & 1 & a & a & 1 & a & 1 & a\end{array}$

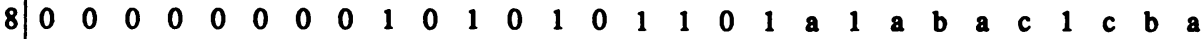

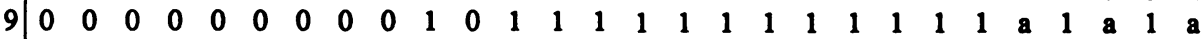

$100 \begin{array}{llllllllllllllllllllllllllll}0 & 0 & 0 & 0 & 0 & 0 & 0 & 0 & 0 & 0 & 1 & 0 & 1 & 0 & 1 & 1 & 0 & 1 & a & 1 & a & 1 & a & a & 1 & a & 1 & a\end{array}$

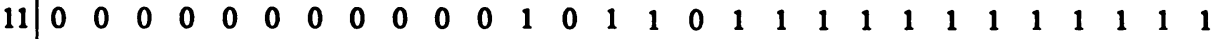

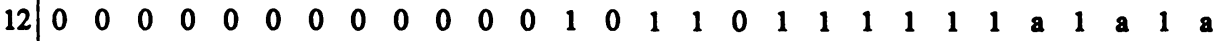

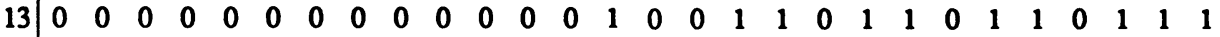

$14 \quad \begin{array}{llllllllllllllllllllllllllll}0 & 0 & 0 & 0 & 0 & 0 & 0 & 0 & 0 & 0 & 0 & 0 & 0 & 0 & 1 & 0 & 0 & 1 & 1 & 1 & 1 & 1 & 1 & 1 & 1 & 1 & 1 & 1\end{array}$

$15 \quad 0 \begin{array}{llllllllllllllllllllllllllll} & 0 & 0 & 0 & 0 & 0 & 0 & 0 & 0 & 0 & 0 & 0 & 0 & 0 & 0 & 1 & 0 & 0 & 1 & 0 & 1 & 1 & 1 & a & 1 & \text { a } & 1 & \text { a }\end{array}$

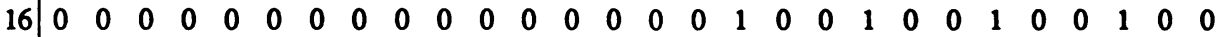

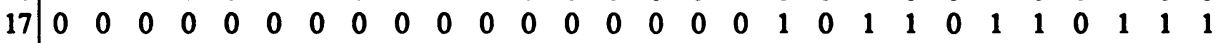

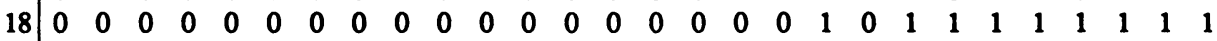

$190 \begin{array}{llllllllllllllllllllllllllll}0 & 0 & 0 & 0 & 0 & 0 & 0 & 0 & 0 & 0 & 0 & 0 & 0 & 0 & 0 & 0 & 0 & 0 & 0 & 1 & 0 & 0 & 1 & 0 & 0 & 1 & 0 & 0\end{array}$

$20 \begin{array}{llllllllllllllllllllllllllll}20 & 0 & 0 & 0 & 0 & 0 & 0 & 0 & 0 & 0 & 0 & 0 & 0 & 0 & 0 & 0 & 0 & 0 & 0 & 0 & 1 & 0 & 1 & 1 & 0 & 1 & 1 & 1\end{array}$

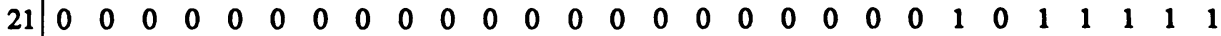

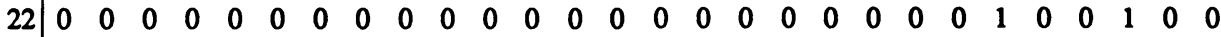

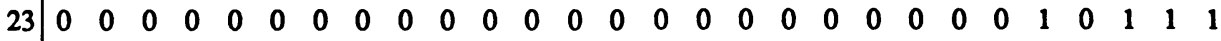

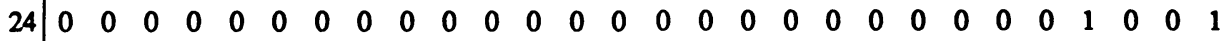

$250 \begin{array}{llllllllllllllllllllllllllll} & 0 & 0 & 0 & 0 & 0 & 0 & 0 & 0 & 0 & 0 & 0 & 0 & 0 & 0 & 0 & 0 & 0 & 0 & 0 & 0 & 0 & 0 & 0 & 0 & 1 & 0 & 0\end{array}$

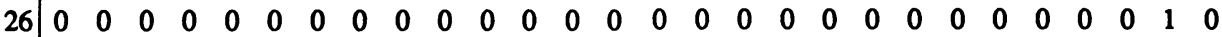

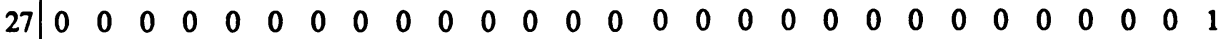

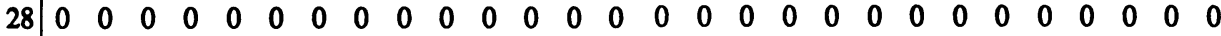

$290 \begin{array}{llllllllllllllllllllllllllll} & 0 & 0 & 0 & 0 & 0 & 0 & 0 & 0 & 0 & 0 & 0 & 0 & 0 & 0 & 0 & 0 & 0 & 0 & 0 & 0 & 0 & 0 & 0 & 0 & 0 & 0 & 0\end{array}$

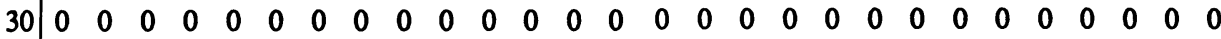

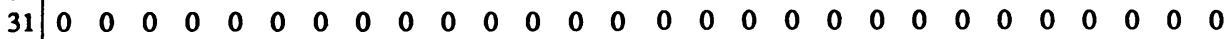

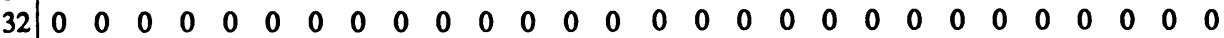

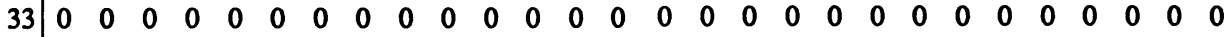

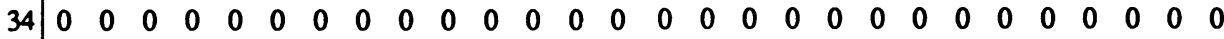

$350 \begin{array}{llllllllllllllllllllllllllll} & 0 & 0 & 0 & 0 & 0 & 0 & 0 & 0 & 0 & 0 & 0 & 0 & 0 & 0 & 0 & 0 & 0 & 0 & 0 & 0 & 0 & 0 & 0 & 0 & 0 & 0 & 0\end{array}$

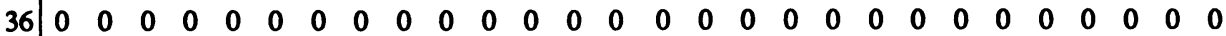

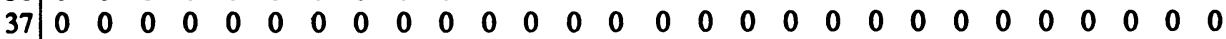

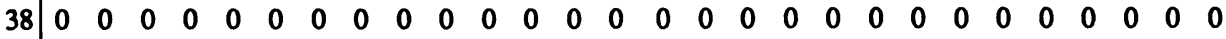

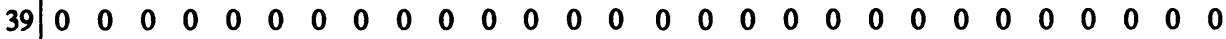

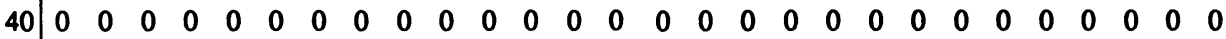
$41 \mid \begin{array}{llllllllllllllllllllllllllll} & 0 & 0 & 0 & 0 & 0 & 0 & 0 & 0 & 0 & 0 & 0 & 0 & 0 & 0 & 0 & 0 & 0 & 0 & 0 & 0 & 0 & 0 & 0 & 0 & 0 & 0 & 0\end{array}$

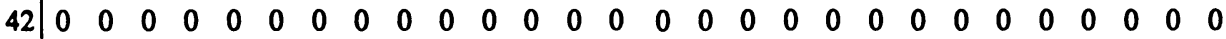
$430 \begin{array}{llllllllllllllllllllllllllll} & 0 & 0 & 0 & 0 & 0 & 0 & 0 & 0 & 0 & 0 & 0 & 0 & 0 & 0 & 0 & 0 & 0 & 0 & 0 & 0 & 0 & 0 & 0 & 0 & 0 & 0 & 0\end{array}$

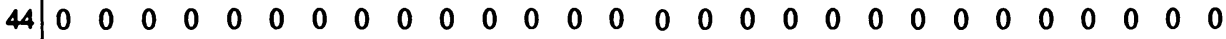
$450 \begin{array}{llllllllllllllllllllllllllll}0 & 0 & 0 & 0 & 0 & 0 & 0 & 0 & 0 & 0 & 0 & 0 & 0 & 0 & 0 & 0 & 0 & 0 & 0 & 0 & 0 & 0 & 0 & 0 & 0 & 0 & 0 & 0\end{array}$

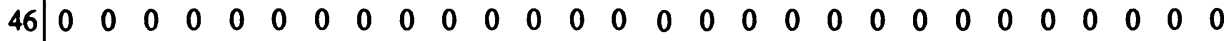

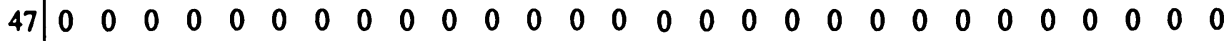

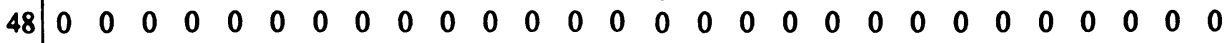
49. $\begin{array}{llllllllllllllllllllllllllll}0 & 0 & 0 & 0 & 0 & 0 & 0 & 0 & 0 & 0 & 0 & 0 & 0 & 0 & 0 & 0 & 0 & 0 & 0 & 0 & 0 & 0 & 0 & 0 & 0 & 0 & 0 & 0\end{array}$ 50 $\begin{array}{llllllllllllllllllllllllllll}0 & 0 & 0 & 0 & 0 & 0 & 0 & 0 & 0 & 0 & 0 & 0 & 0 & 0 & 0 & 0 & 0 & 0 & 0 & 0 & 0 & 0 & 0 & 0 & 0 & 0 & 0 & 0\end{array}$

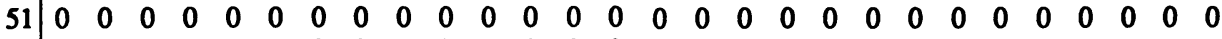

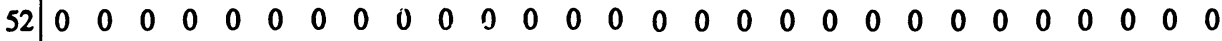
$5330 \begin{array}{llllllllllllllllllllllllllll} & 0 & 0 & 0 & 0 & 0 & 0 & 0 & 0 & 0 & 0 & 0 & 0 & 0 & 0 & 0 & 0 & 0 & 0 & 0 & 0 & 0 & 0 & 0 & 0 & 0 & 0 & 0\end{array}$

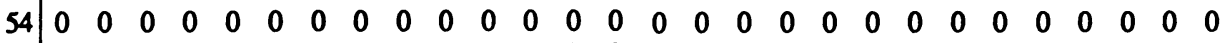

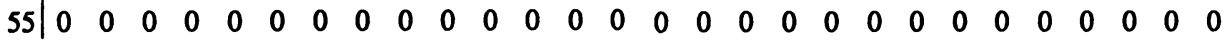


TABLE 3. Kazhdan-Lusztig polynomials for $E_{7}$ (continued)

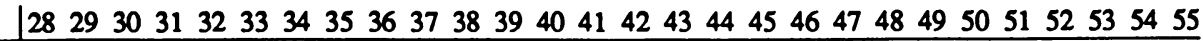

\begin{tabular}{lllllllllllllllllllllllllllll}
\hline & $t$ & $t$ & $y$ & $m$ & $A$ & $D$ & $y$ & $E$ & $G$ & $F$ & $J$ & $M$ & $B$ & $K$ & $N$ & $L$ & $P$ & $H$ & $Q$ & $d$ & $R$ & r & $m$ & y & $D$ & $G$ & $M$ & 1
\end{tabular}

$\begin{array}{lllllllllllllllllllllllllllll}1 & t & t & g & m & A & f & y & E & d & F & J & 1 & B & K & N & L & P & H & Q & d & R & r & m & y & D & G & j & 1\end{array}$

$\begin{array}{lllllllllllllllllllllllllllll}2 & g & t & g & m & w & f & y & v & d & F & r & 1 & B & K & a & L & P & H & Q & d & R & \text { r } & m & y & D & q & j & 1\end{array}$

$\begin{array}{lllllllllllllllllllllllllllll}3 & g & c & g & m & w & f & y & v & d & z & r & 1 & B & x & a & L & c & H & Q & d & R & r & m & y & s & q & j & 1\end{array}$

$\begin{array}{lllllllllllllllllllllllllllll}4 & g & c & c & m & i & f & n & v & d & z & r & 1 & B & x & a & C & c & H & g & d & R & r & m & t & s & q & j & 1\end{array}$

$\begin{array}{lllllllllllllllllllllllllllll}5 & \text { a } & c & c & 1 & i & f & n & v & d & z & \text { r } & 1 & B & x & \text { a } & C & c & f & g & d & t & \text { r } & b & t & s & q & j & 1\end{array}$

$\begin{array}{lllllllllllllllllllllllllllllllllll}6 & g & c & c & m & i & b & n & g & d & p & r & 1 & b & x & a & C & c & H & g & d & t & e & m & t & s & q & j & 1\end{array}$

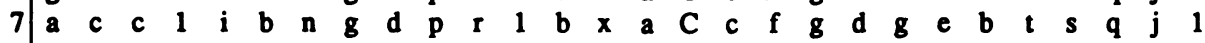

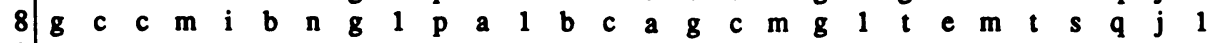

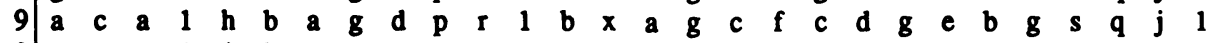

$\begin{array}{lllllllllllllllllllllllllllll}10 & \text { a } & c & c & 1 & \mathrm{i} & \mathrm{b} & \mathrm{n} & \mathrm{g} & 1 & \mathrm{p} & \mathrm{a} & 1 & \mathrm{~b} & \mathrm{c} & \mathrm{a} & \mathrm{g} & \mathrm{c} & \mathrm{b} & \mathrm{g} & 1 & \mathrm{~g} & \mathrm{e} & \mathrm{b} & \mathrm{t} & \mathrm{s} & \mathrm{q} & \mathrm{j} & 1\end{array}$

$\begin{array}{lllllllllllllllllllllllllllll}11 & a & 1 & a & 1 & h & b & a & g & d & c & r & 1 & b & e & a & g & a & f & c & d & g & e & b & g & f & q & j & 1\end{array}$

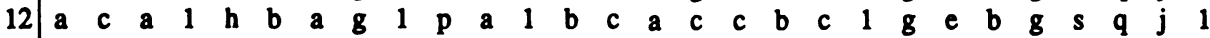

$\begin{array}{lllllllllllllllllllllllllllll}13 & 1 & 1 & a & 1 & a & b & a & b & d & c & d & 1 & b & e & 1 & g & a & f & c & d & g & e & b & g & f & d & j & 1\end{array}$

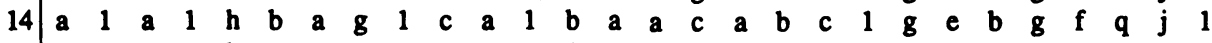

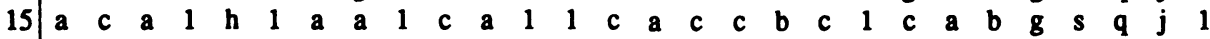

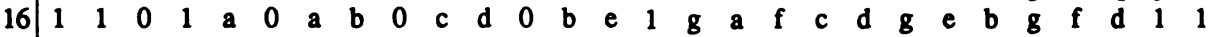

$\begin{array}{lllllllllllllllllllllllllllll}17 & 1 & 1 & a & 1 & a & b & a & b & 1 & c & 1 & 1 & b & a & 1 & c & a & b & c & 1 & g & e & b & g & f & d & j & 1\end{array}$

$\begin{array}{lllllllllllllllllllllllllllll}18 & \text { a } & 1 & \text { a } & 1 & \text { h } & 1 & \text { a } & \text { a } & 1 & \text { a } & \text { a } & 1 & 1 & \text { a } & \text { a } & \text { c } & \text { a } & \text { b } & \text { c } & 1 & \text { c } & \text { a } & \text { b } & \text { g } & f & \text { q } & j & 1\end{array}$

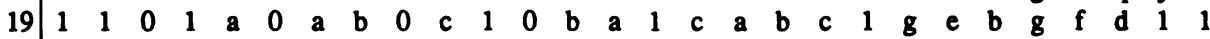

$\begin{array}{lllllllllllllllllllllllllllll}20 & 1 & 1 & a & 1 & a & 1 & a & 1 & 1 & a & 1 & 1 & 1 & a & 1 & c & a & b & c & 1 & c & a & b & g & f & d & j & 1\end{array}$

$\begin{array}{lllllllllllllllllllllllllllll}21 & a & 1 & 1 & 1 & a & 1 & 1 & a & 1 & a & a & 1 & 1 & a & a & a & a & b & a & 1 & c & a & b & c & f & q & j & 1\end{array}$

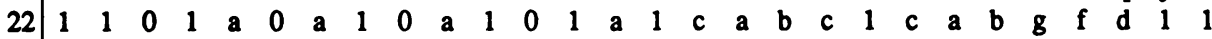

$\begin{array}{lllllllllllllllllllllllllllll}23 & 1 & 1 & 1 & 1 & 1 & 1 & 1 & 1 & 1 & \text { a } & 1 & 1 & 1 & \text { a } & 1 & \text { a } & \text { a } & \text { b } & \text { a } & 1 & c & \text { a } & \text { b } & \text { c } & f & d & j & 1\end{array}$

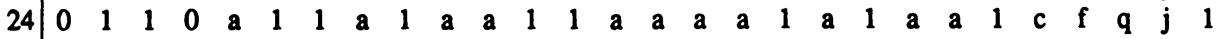

$\begin{array}{lllllllllllllllllllllllllllll}25 & 1 & 1 & 0 & 1 & 1 & 0 & 1 & 1 & 0 & \text { a } & 1 & 0 & 1 & \text { a } & 1 & \text { a } & \text { a } & \text { b } & \text { a } & 1 & c & \text { a } & \text { b } & \text { c } & \text { f } & d & 1 & 1\end{array}$

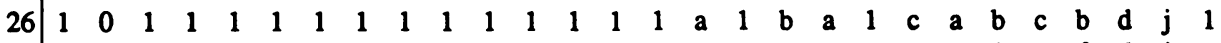

$\begin{array}{lllllllllllllllllllllllllllll}27 & 0 & 1 & 1 & 0 & 1 & 1 & 1 & 1 & 1 & a & 1 & 1 & 1 & a & 1 & a & a & 1 & a & 1 & a & a & 1 & c & f & d & j & 1\end{array}$

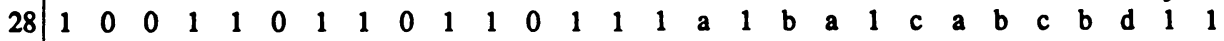

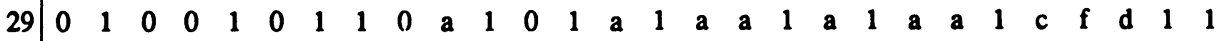

$30 \begin{array}{llllllllllllllllllllllllllll}30 & 0 & 1 & 0 & 1 & 1 & 1 & 1 & 1 & 1 & 1 & 1 & 1 & 1 & 1 & \text { a } & 1 & 1 & \text { a } & 1 & \text { a } & \text { a } & 1 & \text { c } & \text { b } & d & j & 1\end{array}$

$\begin{array}{lllllllllllllllllllllllllllll}31 & 0 & 0 & 0 & 1 & 0 & 0 & 1 & 0 & 0 & 1 & 0 & 0 & 1 & 1 & 0 & \text { a } & 1 & \text { b } & \text { a } & 1 & c & a & b & c & b & 1 & 1 & 1\end{array}$

$32 \quad 0 \begin{array}{llllllllllllllllllllllllllll} & 0 & 0 & 0 & 1 & 0 & 1 & 1 & 0 & 1 & 1 & 0 & 1 & 1 & 1 & a & 1 & 1 & a & 1 & a & a & 1 & c & b & d & 1 & 1\end{array}$

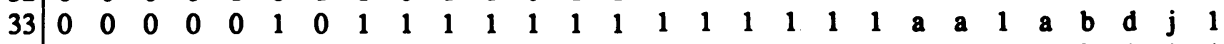

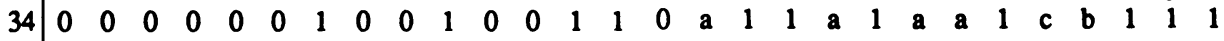

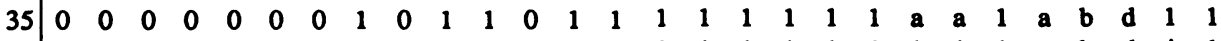

$36 \quad \begin{array}{llllllllllllllllllllllllllll}0 & 0 & 0 & 0 & 0 & 0 & 0 & 0 & 1 & 0 & 1 & 1 & 0 & 1 & 1 & 1 & 1 & 1 & 1 & 1 & 1 & 1 & 1 & a & b & d & j & 1\end{array}$

37 $\quad \begin{array}{llllllllllllllllllllllllllll}0 & 0 & 0 & 0 & 0 & 0 & 0 & 0 & 0 & 1 & 0 & 0 & 1 & 1 & 0 & 1 & 1 & 1 & 1 & 1 & a & a & 1 & a & b & 1 & 1 & 1\end{array}$

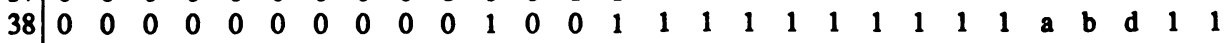

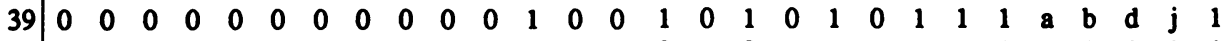

$40 \begin{array}{llllllllllllllllllllllllllll}4 & 0 & 0 & 0 & 0 & 0 & 0 & 0 & 0 & 0 & 0 & 0 & 1 & 0 & 0 & 1 & 0 & 1 & 1 & 1 & \text { a } & \text { a } & 1 & \text { a } & 1 & 1 & 1 & 1\end{array}$

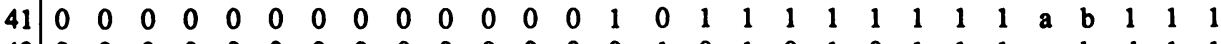

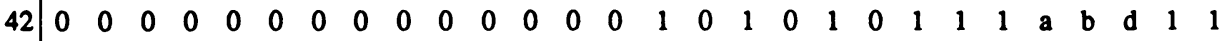

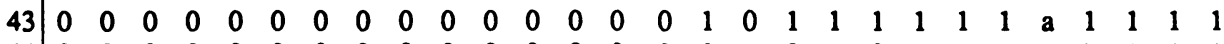

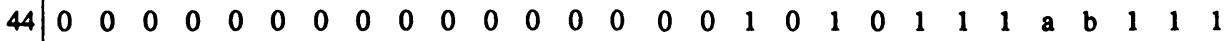

$45 \mid \begin{array}{llllllllllllllllllllllllllll}0 & 0 & 0 & 0 & 0 & 0 & 0 & 0 & 0 & 0 & 0 & 0 & 0 & 0 & 0 & 0 & 0 & 1 & 0 & 1 & 1 & 1 & 1 & 1 & 1 & 1 & 1 & 1\end{array}$

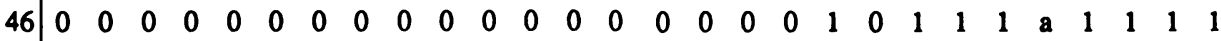

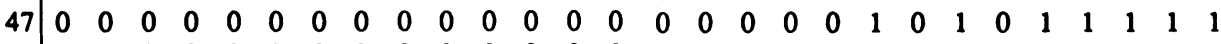

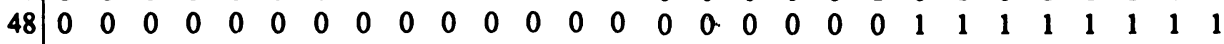

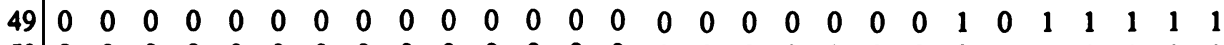

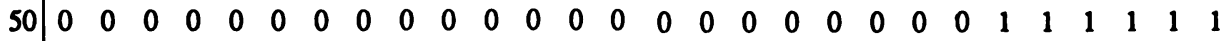

$\begin{array}{lllllllllllllllllllllllllllll}51 & 0 & 0 & 0 & 0 & 0 & 0 & 0 & 0 & 0 & 0 & 0 & 0 & 0 & 0 & 0 & 0 & 0 & 0 & 0 & 0 & 0 & 0 & 0 & 1 & 1 & 1 & 1 & 1\end{array}$

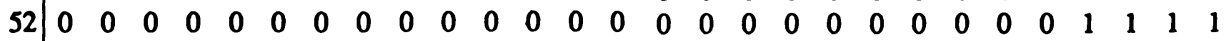

$53\left(\begin{array}{llllllllllllllllllllllllllll} & 0 & 0 & 0 & 0 & 0 & 0 & 0 & 0 & 0 & 0 & 0 & 0 & 0 & 0 & 0 & 0 & 0 & 0 & 0 & 0 & 0 & 0 & 0 & 0 & 1 & 1 & 1\end{array}\right.$

$54\left(\begin{array}{llllllllllllllllllllllllllll}5 & 0 & 0 & 0 & 0 & 0 & 0 & 0 & 0 & 0 & 0 & 0 & 0 & 0 & 0 & 0 & 0 & 0 & 0 & 0 & 0 & 0 & 0 & 0 & 0 & 0 & 1 & 1\end{array}\right.$

55 $\begin{array}{llllllllllllllllllllllllllll}0 & 0 & 0 & 0 & 0 & 0 & 0 & 0 & 0 & 0 & 0 & 0 & 0 & 0 & 0 & 0 & 0 & 0 & 0 & 0 & 0 & 0 & 0 & 0 & 0 & 0 & 0 & 1\end{array}$ 
when reproducing these matrices. The identifications employed are given in Table 1. The K-L polynomials for $E_{6}$ and $E_{7}$ are given in Tables 2 and 3, respectively. There, $P_{y, w}$ is the entry in the row indexed by $y$ and the column indexed by $w$; we use the indexing for $W^{K}$ defined in $[\mathbf{1}, \mathbf{2}$, or $\mathbf{6}]$. (See note added in proof.)

7. Applications. In this section, we apply the above results to determine certain coefficients of the Kazhdan-Lusztig polynomials for Hermitian symmetric spaces. We first show that the leading coefficient of $P_{y, w}(u)$ is always 1 (if $y \leq$ $w)$. For $y<w$, it is known [10] that $P_{y, w}(u)$ is a polynomial of degree at most $(l(y)-l(w)-1) / 2$. Define $\mu(y, w)$ to be the coefficient of this term of highest possible degree when $y<w$, and 0 otherwise. We determine, for $G=\operatorname{Sp}(n, \mathbf{R})$ or $\mathrm{SO}^{*}(2 n)$, the pairs $y<w$ for which $\mu(y, w) \neq 0$ (and hence $=1$, by the first result).

(7.1) Proposition. Let $G$ be one of the groups in (1.1), $\mathfrak{g}$ its complexified Lie algebra, and $\mathfrak{p}$ the associated parabolic subalgebra (cf. §2). Let $y, w \in W^{K}$ with $y \leq w$. Then the leading coefficient of $P_{y, w}(u)$ is 1 .

PrOOF. This was observed for $G=\mathrm{SU}(p, q)$ by Lascoux-Schützenberger in [11]. For $G=\mathrm{SO}(n, \mathbf{R})$ the result is clear from Proposition 5.1, and for $G$ exceptional, it follows by inspection of Table 1.

Since the K-L polynomials for $\mathrm{SO}^{*}(2(n+1))$ and for $\mathrm{Sp}(n, \mathbf{R})$ are identical, we may assume that $G=\operatorname{Sp}(n, \mathbf{R})$. Now every edge of the tree $A(w / y)$ can be (independently) labelled with its "capacity," except possibly certain edges having both odd capacity and predecessors (cf. (3.10)). We claim, nevertheless, that there is a unique labelling $v$ having maximal weight $|v|$, and hence that $P_{y, w}(u)=u^{|v|}+$ lower degree terms. Define $v$ by labelling the edges of $A(w / y)$ from bottom to top, assigning labels to preceding edges before succeeding edges, and giving each edge the largest label allowed by (3.10). It is clear that $|v|$ is maximal. We must show that there is no other labelling $v^{\prime}$ with $\left|v^{\prime}\right|=|v|$. There are three possible ways this might fail.

Case (i). $v^{\prime}$ is obtained from $v$ by decreasing the label on some "initial edge" (one having no predecessors) by two, thereby allowing the labels on two succeeding edges to increase by one. Denote the capacity of the initial edge (which must correspond to a terminal $\alpha$ or an $\alpha \alpha$-pair in $w$ ) by $c_{0}$, and the capacities of the other two edges by $c_{1}$ and $c_{2}$ (with edge " 0 " preceding edge " 1 " preceding edge "2"). Without loss of generality, we may assume $c_{0}$ is even. The hypothesis implies that $c_{1}$ and $c_{2}$ must both be odd, and the relevant labels in $v$ are $c_{0}, c_{1}-1$, and $c_{2}-1$. The corresponding labels in $v^{\prime}$ are $c_{0}-2, c_{1}$ and $c_{2}$. We conclude that $c_{1}<c_{0}$ and $c_{2}<c_{1}-1$, while $c_{1} \geq c_{0}-2$ and $c_{2} \geq c_{1}$. This is a contradiction.

Case (ii). $v^{\prime}$ is obtained from $v$ by decreasing the label on some initial edge by two, thereby allowing the labels on one succeeding edge and one higher edge to increase by one. In this case, the higher edge must correspond to an $\alpha \alpha$-pair in $w$, and must lie above both the other two edges. Denote by $c_{0}$ the capacity of the initial edge, and by $c_{1}$ that of the succeeding edge. We may assume $c_{0}$ is even, and as in case (i), $c_{1}$ must be odd. In fact, it follows as above that $c_{1}=c_{0}-1$. The relevant labels in $v$ must be $c_{0}, c_{1}-1$, and $c_{1}-1$; and in $v^{\prime}, c_{0}-2, c_{1}$ and $c_{1}$. But since $c_{1}=c_{0}-1>c_{0}-2$, this contradicts the requirement that the labels be nonincreasing from bottom to top. 
Case (iii). $v^{\prime}$ is obtained from $v$ by decreasing the label on some noninitial edge by one, thereby allowing the label on some succeeding edge to increase by one. Denote by $c_{1}$ the capacity of the preceding edge, and by $c_{2}$ that of the succeeding edge. Then $c_{2}$ must be odd, and the labels on edge " 1 " and edge "2" (respectively) are: $c_{2}$ and $c_{2}-1$ in $v ; c_{2}-1$ and $c_{2}$ in $v^{\prime}$. On the other hand, we claim that the label $\left(c_{2}\right)$ on edge " 1 " in $v$ must be less than or equal to the labels on all edges preceding it. If not, then the label on edge " 2 " in $v$ would not be forced to be $c_{2}-1$, but could also be $c_{2}$. In particular, $c_{2}$ must be even (by (3.10)), a contradiction. Q.E.D.

(7.2) Proposition. Let $G=\operatorname{Sp}(n, \mathbf{R})$ or $\mathrm{SO}^{*}(2(n+1))$. For $y, w \in W^{K}$ $\mu(y, w) \neq 0$ if and only if one of the following holds:

(i) $w=w^{\prime} \beta z \alpha w^{\prime \prime}, y=w^{\prime} \alpha z \beta w^{\prime \prime}$ with $z \in Z$;

(ii) $w=w^{\prime} \beta z, y=w^{\prime} \alpha z$ with $z \in Z$; or

(iii) $w=w^{\prime} \beta z \beta z^{\prime}, y=w^{\prime} \alpha z \alpha z^{\prime}$ with $z \in Z$, and $z^{\prime}$ consisting entirely of linked $\alpha \beta$-pairs, linked $\alpha \alpha$-pairs, and exactly one terminal $\alpha$.

If any of these conditions hold, then $\mu(y, w)=1$.

ProOF. The last statement will follow from the first and Proposition 7.1.

Note that if $\mu(y, w) \neq 0$ and $l(y)-l(w)>1$, it follows from Lemma 3.5 that $y$ is normalized with respect to $w$ (cf. (3.6)). We proceed in steps to obtain from the pair $(y, w)$ a "reduced pair" $\left(y^{\text {red }}, w^{\text {red }}\right)$.

Step 1. If possible, choose a factor $\alpha \beta$ in $y$, so that $y=y^{\prime} \alpha \beta y^{\prime \prime}, w=w^{\prime} \alpha \beta w^{\prime \prime}$, for some $y^{\prime}, y^{\prime \prime}, w^{\prime}, w^{\prime \prime}$ with $\left|y^{\prime}\right|=\left|w^{\prime}\right|$. Let $c$ be the capacity of the corresponding minimal edge. Then by (3.14), $\operatorname{deg} P_{y, w}=c+\operatorname{deg} P_{y^{\prime} y^{\prime \prime}, w^{\prime} w^{\prime \prime}}$. And by the proof of $(3.13), 2 c=[l(y)-l(w)]-\left[l\left(y^{\prime} y^{\prime \prime}\right)-l\left(w^{\prime} w^{\prime \prime}\right)\right]$. Thus $\mu(y, w) \neq 0$ if and only if $\mu\left(y^{\prime} y^{\prime \prime}, w^{\prime} w^{\prime \prime}\right) \neq 0$. In particular, $y^{\prime} y^{\prime \prime}$ must be normalized with respect to $w^{\prime} w^{\prime \prime}$ (unless their lengths differ by 1 ).

Continue to remove adjacent factors $\alpha \beta$, eventually arriving at a pair $\left(y^{0}, w^{0}\right)$ with $\mu\left(y^{0}, w^{0}\right) \neq 0$, such that either $y^{0}$ contains no factor $\alpha \beta$, or else $y^{0}$ does contain an $\alpha \beta$ factor and $l\left(y^{0}\right)-l\left(w^{0}\right)=1$. In the latter case, we must have $y^{0}=x^{\prime} \alpha \beta x^{\prime \prime}$, $w^{0}=x^{\prime} \beta \alpha x^{\prime \prime}$ for some $x^{\prime}, x^{\prime \prime}$, and thus $y, w$ are as in case (i).

Step 2. Assume $l\left(y^{0}\right)-l\left(w^{0}\right)>1$, and suppose $y^{0}$ ends in $\alpha$. Then $y^{0}=u \sigma \alpha$, $w^{0}=v \tau \alpha$ for some $u, v$ with $|u|=|v|$. Let $c$ be the capacity of the terminal $\alpha$ edge. Then by (3.14), $c$ must be even, and $\operatorname{deg} P_{y, w}=c+\operatorname{deg} P_{u, v}$. Again, the proof of (3.13) implies that $2 c=[l(y)-l(w)]-[l(u)-l(v)]$. Hence $\mu\left(y^{0}, w^{0}\right) \neq 0$ if and only if $\mu(u, v) \neq 0$. If $u$ ends in $\alpha$, then $\sigma=\alpha$, since by assumption $y^{0}$ contains no $\alpha \beta$ factors. If, in addition, $l(u)-l(v)>1$, then $\tau=\alpha$, otherwise the capacity of the terminal $\alpha$ edge of $A(v / u)$ would be odd.

Repeat Step 2, and continue in this fashion. Eventually one arrives at a pair $y^{\text {red }} \leq w^{\text {red }}$ with $\mu\left(y^{\text {red }}, w^{\text {red }}\right) \neq 0$ such that $y^{\text {red }}$ contains no $\alpha \beta$ factors, and either $y^{\text {red }}$ does not end in $\alpha$, or else $y^{\text {red }}$ does end in $\alpha$ and $l\left(y^{\text {red }}\right)-l\left(w^{\text {red }}\right)=1$. In the former case, $y^{\text {red }}=\beta \beta \cdots \beta$, forcing $w^{\text {red }}=\beta \beta \cdots \beta$, hence $y=w$. But this contradicts $\mu(y, w) \neq 0$. So we must be in the latter case, with $y^{\text {red }}=x \alpha$, $w^{\text {red }}=x \beta$ for some $x$. This leads to two possibilities for $(y, w)$ : either $y=w^{\prime} \alpha z$, $w=w^{\prime} \beta z$ with $z \in Z$; or $y=w^{\prime} \alpha z \alpha z^{\prime}, w=w^{\prime} \beta z \tau z^{\prime}$, with $z \in Z$ and $z^{\prime}$ as in Case (iii). The first possibility is just Case (ii). In the second situation, we must have $\tau=\beta$, for otherwise the next-to-last application of Step 2 would be applied to the 
pair $(x \alpha \alpha \alpha, x \beta \alpha \alpha)$ with terminal $\alpha$ capacity $=1$, which is impossible. Thus we are in case (iii). Q.E.D.

NOTE ADDED IN PROOF. Since this paper was written, the K-L polynomials for the Hermitian symmetric $E_{6}$ and $E_{7}$ have been independently checked, using a computer program written by the author and based on the recursion relations of Deodhar [7].

\section{REFERENCES}

1. B. Boe and D. H. Collingwood, Intertwining operators between holomorphically induced modules, Pacific J. Math. 124 (1986), 73-84.

2. B. Boe, T. J. Enright and B. Shelton, Determination of the intertwining operators for holomorphically induced representations of Hermitian symmetric pairs, Pacific J. Math. 131 (1988), 39-50.

3. W. Borho and J. C. Jantzen, Über primative Ideale in der Einhüllenden einer halbeinfachen Lie-Algebra, Invent. Math. 39 (1977), 1-53.

4. L. Casian and D. H. Collingwood, The Kazhdan-Lusztig conjecture for generalized Verma modules, Math. Z. 195 (1987), 581-600.

5. D. H. Collingwood, The n-homology of Harish-Chandra modules: generalizing a theorem of Kostant, Math. Ann. 272 (1985), 161-187.

6. D. H. Collingwood, R. S. Irving and B. Shelton, Filtrations on generalized Verma modules for Hermitian symmetric pairs, J. Reine Angew. Math. 383 (1988), 54-86.

7. V. V. Deodhar, On some geometric aspects of Bruhat orderings. II: the parabolic analogue of Kazhdan-Lusztig polynomials, J. Algebra 111 (1987), 483-506.

8. T. J. Enright and B. Shelton, Categories of highest weight modules: applications to classical Hermitian symmetric pairs, Mem. Amer. Math. Soc., vol. 367, 1987.

9. H. Hiller, Geometry of Coxeter groups, Research Notes in Math., Pitman, Boston-LondonMelbourne, 1982.

10. D. Kazhdan and G. Lusztig, Representations of Coxeter groups and Hecke algebras, Invent. Math. 53 (1979), 165-184.

11. A. Lascoux and M.-P. Schützenberger, Polynômes de Kazhdan et Lusztig pour les grassmanniennes, Astérisque 87-88 (1981), 249-266.

Department of Mathematics, University of Georgia, Athens, Georgia 30602 